\title{
Systematic Efficient Parallelization of Scan and Other List Homomorphisms
}

\author{
Sergei Gorlatch ${ }^{\star}$ \\ University of Passau, D-94030 Passau, Germany
}

\begin{abstract}
Homomorphisms are functions which can be parallelized by the divide-and-conquer paradigm. A class of distributable homomorphisms (DH) is introduced and an efficient parallel implementation schema for all functions of the class is derived by transformations in the Bird-Meertens formalism. The schema can be directly mapped on the hypercube with an unlimited or an arbitrary fixed number of processors, providing provable correctness and predictable performance. The popular scan-function (parallel prefix) illustrates the presentation: the systematically derived implementation for scan coincides with the practically used "folklore" algorithm for distributed-memory machines.
\end{abstract}

\section{Introduction}

This paper deals with formal design of parallel programs. We advocate that the issues of correctness and performance should and can be addressed during the design/derivation process, rather than as an afterthought.

As a derivational calculus we use the Bird-Meertens Formalism (BMF) [1]. Computations are specified using a set of higher-order functions over lists and other data structures; the specification is refined into an executable form by semantically sound transformation rules, which guarantees the correctness of the target program. We structure the derivation process, by exposing the points where the design decisions are made and estimating the target performance. Such a structuring naturally leads to extracting the typically used and efficiently implementable classes (templates, skeletons) of parallelism.

In this paper, we study functions, called homomorphisms, which are parallelizable using the important divide-and-conquer paradigm. We define a class, called Distributable Homomorphisms (DH), and derive an efficient and provably correct parallel implementation schema for all functions of the class.

As an illustrating example, we use the scan (parallel prefix) function, which encapsulates a computational pattern common for many parallel applications [2]. The specialization of our parallel implementation schema for the case of scan yields the known parallel algorithms for scan on a hypercube with either a linear or an arbitrary fixed number of processors. In contrast to the usual ad hoc presentation of these algorithms, we derive them in a systematic sequence of design steps, which are methodologically substantiated and formally correct.

\footnotetext{
* The author was partially supported by the DAAD cooperation programs ARC and PROCOPE, and by the Project INTAS-93-1702.
} 


\section{BMF, Homomorphisms and Scan}

We use a variant of the Bird-Meertens Formalism (BMF) with non-empty lists of length $2^{k}, k=0,1, \cdots$ (powerlists [3]). Function length yields the length of a list. The constructors are: (i) [.] yielding the singleton list and (ii) balanced concatenation $\#$, where $x+y$ is defined iff length $x=$ length $y=2^{k}$.

We use the following functions and functionals, defined informally:

- backward functional composition;

id the identity function;

map $f \quad$ map of an unary function $f$, i.e., $\operatorname{map} f\left[x_{1}, \cdots, x_{n}\right]=\left[f x_{1}, \cdots, f x_{n}\right]$;

red $(\odot)$ balanced reduce with a binary associative operation $\odot$, where $\operatorname{red}(\odot)[a]=a, \operatorname{red}(\odot)(x+y)=(\operatorname{red}(\odot) x) \odot(\operatorname{red}(\odot) y) ;$

zip $(\odot)$ component-wise application of $\odot$ to a pair of lists of equal length: $z i p(\odot)\left(\left[x_{1}, \cdots, x_{n}\right],\left[y_{1}, \cdots, y_{n}\right]\right)=\left[\left(x_{1} \odot y_{1}\right), \cdots,\left(x_{n} \odot y_{n}\right)\right]$

$\left\langle>\quad\right.$ "zipped tupling": for a tuple of functions $f_{i}:[\alpha] \rightarrow[\alpha], i=1, \cdots n$, function $\left\langle f_{1}, \cdots, f_{n}\right\rangle$ yields the list of result tuples.

Definition 1. A list function $h$ is a homomorphism iff there exists a binary associative combine operator $\oslash$, such that for all lists $x$ and $y$ :

$$
h(x+y)=h(x) \oslash h(y)
$$

i.e., the value of $h$ on a list depends in a particular way (using $\oslash$ ) upon the values of $h$ on the pieces of the list.

The computations of $h(x)$ and $h(y)$ are independent and can be carried out in parallel, which expresses the well-known divide-and-conquer paradigm.

Theorem 2 (Bird [1]). Function $h$ is a homomorphism iff:

$$
h=\operatorname{red}(\oslash) \circ \operatorname{map}(f)
$$

where $\oslash$ is from (1) and $f(a)=h([a])$.

Theorem 2 provides a common parallelization for all homomorphisms as a composition of two stages [4]: the first, map, is totally parallel, the second, red, can be parallelized on a tree-like structure, with $\oslash$ applied in the nodes.

There are two problems for a given function: first, how to find the combine operator $\oslash$ of (2) and, second, how to implement the red stage efficiently in parallel. In [5], we described a systematic approach to constructing the combine operator, starting from two sequential representations of the given function. The present paper deals with the second problem, the implementation.

For a homomorphism of type $[\alpha] \rightarrow \alpha$, there are a logarithmic number of steps in the tree computation, with communications of constant size, which yields an efficient algorithm. However, when a homomorphism yields a list, i.e., its combine operator contains $\#$, then the tree implementation requires linear execution time, independently of the number of processors [6]. 
Scan as a Homomorphism. Our illustrating example is the scan-function which, for associative $\odot$ and a list, computes "prefix sums", e.g.:

$$
\operatorname{scan}(\odot)[a, b, c, d]=[a,(a \odot b),(a \odot b \odot c),(a \odot b \odot c \odot d)]
$$

Function scan is a homomorphism with combine operator $\oslash$ :

$$
\begin{aligned}
& \operatorname{scan}(\odot)(x+y)=S_{1} \oslash S_{2}=S_{1} \#\left(\operatorname{map}\left(\operatorname{last}\left(S_{1}\right) \odot\right) S_{2}\right), \\
& \text { where } S_{1}=\operatorname{scan}(\odot) x, S_{2}=\operatorname{scan}(\odot) y .
\end{aligned}
$$

Here, so-called sectioning $(a \odot)$ is used: $(a \odot) b=a \odot b$.

Despite the fact that $\oslash$ contains $\#$, there exist efficient parallel algorithms for $\operatorname{scan}[7,8]$, which, rather than producing a monolithic output list, distribute it between processors. Our goal is to derive such algorithms systematically.

\section{Distributable Homomorphisms}

We introduce a specific class of homomorphisms by restricting the permitted form of the combine operator.

Definition 3. For two binary associative operations $\oplus$ and $\otimes$ on elements, the combine operator $\oplus \otimes$ on lists is defined as follows:

$$
u \oplus v=z i p(\oplus)(u, v)+z i p(\otimes)(u, v)
$$

We write $\oplus \uparrow \otimes$ for the following homomorphism with combine operator $\oplus \otimes$ :

$$
\begin{aligned}
\oplus \downarrow \otimes[a] & =[a] \\
\oplus \downarrow \otimes(x+y) & =((\oplus \downarrow \otimes) x) \oplus \otimes((\oplus \downarrow \otimes) y)
\end{aligned}
$$

Definition 4. Function $h:[\alpha] \rightarrow[\alpha]$ is a distributable homomorphism (DH) iff $h=\oplus \downarrow \otimes$, for some $\oplus$ and $\otimes$.


Fig. 1. A general homomorphism (left) and a distributable homomorphism (right), computed on a concatenation of two lists

The "distributed reduction": $\operatorname{redd}(\odot) x=[\operatorname{red}(\odot) x, \operatorname{red}(\odot) x, \cdots, \operatorname{red}(\odot) x]$ is obviously a homomorphism with the combine operator:

$$
R_{1} \ominus R_{2}=z i p(\odot)\left(R_{1}, R_{2}\right)+z i p(\odot)\left(R_{1}, R_{2}\right)
$$


This fits format (4), thus redd is a $\mathrm{DH}$ :

$$
\operatorname{redd}(\odot)=\odot \downarrow \odot
$$

Function redd is implemented as ReduceAll in the recent MPI standard [9].

Scan: Adjusting to DH. Let us try to express the right-hand side of (3) in format (4), i.e., with both arguments of $\#$ in zipped form. This is easy for the left argument but requires an additional function for the right argument of $\#$ :

$$
\begin{aligned}
S_{1} & =z i p\left(\pi_{1}\right)\left(S_{1}, S_{2}\right) \\
\operatorname{map}\left(\operatorname{last}\left(S_{1}\right) \odot\right) S_{2} & =z i p(\odot)\left(R_{1}, S_{2}\right)
\end{aligned}
$$

where $\pi_{1}$ yields the first element of a pair and $R_{1}=\operatorname{redd}(\odot) x$. We can thus obtain the desired format if we "tuple" scan together with redd into new function $<$ scan, redd >, which can be adjusted to (4) by transition to lists of pairs.

The target expression of $s c a n$ is as follows:

$$
\begin{aligned}
\operatorname{scan}(\odot)= & \left(\operatorname{map} \pi_{1}\right) \circ(\oplus \uparrow \otimes) \circ \operatorname{map}(\text { pair }) \\
\text { where } & \text { pair } a=(a, a) \\
& \left(s_{1}, r_{1}\right) \oplus\left(s_{2}, r_{2}\right)=\left(s_{1}, r_{1} \odot r_{2}\right) \\
& \left(s_{1}, r_{1}\right) \otimes\left(s_{2}, r_{2}\right)=\left(r_{1} \odot s_{2}, r_{1} \odot r_{2}\right)
\end{aligned}
$$

Operations $\oplus$ and $\otimes$ work on pairs and are read off from (6), (8) and (9).

The systematic adjustment of scan to the DH format yields exactly the pair structure, its initialization by function pair and the computations $\oplus$ and $\otimes$ in (11), which are used ad hoc in the known efficient algorithms for scan.

\section{Towards a Hypercube Implementation}

Our goal is to find a provably correct and efficient parallel implementation for all DH functions. As a particular parallel topology for lists of length $n=2^{k}$, we take a $k$-dimensional hypercube with $n$ nodes. We use the standard encoding: the position $l, 0 \leq l<n$, of the list is stored in the hypercube node, whose index is the $k$-bit representation of $l$. Processor $l$ can communicate with its $k$ neighbours; the neighbour in dimension $d$ is $\operatorname{xor}\left(l, 2^{d-1}\right)$, where $x$ or is the bitwise exclusive OR. So, the only difference between a list of length $n=2^{k}$ and the $k$-dimensional hypercube is that in the latter we have random element access and communication primitives. To make this analogy more visible, we abuse the typing by using $[\alpha]$ for both types.

We introduce a pattern of the hypercube behaviour, skeleton swap, which describes a pair-wise communication in dimension $d$, followed by a computation with $\oplus$ and $\otimes$, such that for an index $l$ of a list $x$ :

$$
\begin{aligned}
\text { swap } d(\oplus, \otimes) x l= & x(l) \oplus x(l+d), & & \text { if } l<x o r\left(l, 2^{d-1}\right) \\
& x(l-d) \otimes x(l), & & \text { otherwise }
\end{aligned}
$$

where length $(x)=2^{k}, 1 \leq d \leq k, 0 \leq l<2^{k}$.

Let us define $\operatorname{swap}^{k}=(\operatorname{swap} k) \circ \cdots(\operatorname{swap} 2) \circ(\operatorname{swap} 1)$. 
Proposition 5. Every DH function is implementable as follows:

$$
\oplus \downarrow \otimes=\operatorname{swap}^{k}(\oplus, \otimes)
$$

Scan: the Hypercube Implementation. Implementation (12) is of a general nature; for a particular function, it suffices to substitute concrete operations for $\oplus$ and $\otimes$. From (10) and (12) we obtain the following program for scan:

$$
\operatorname{scan}(\odot)=\operatorname{map}\left(\pi_{1}\right) \circ \operatorname{swap}^{k}(\oplus, \otimes) \circ \operatorname{map}(\text { pair })
$$

where pair, $\oplus$ and $\otimes$ are defined by (11).

This is the well-known "folklore" implementation [7]. In Figure 2, it is illustrated on the 2-dimensional hypercube which is computing $\operatorname{scan}(+)[1,2,3,4]$.



map (pair)

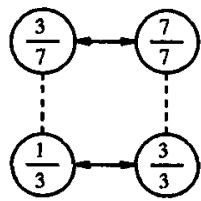

swap 1



swap 2

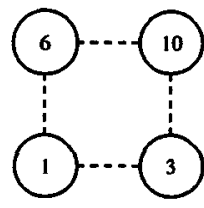

$\operatorname{map}\left(\pi_{1}\right)$

Fig. 2. Computing scan on a hypercube

The time complexity of computing scan for a list of length $n$, on $n$ processors, is $O(\log n)$. The cost (time-processor product [7]) is $O(n \log n)$, whereas the cost of the sequential computation is $O(n)$. So the implementation is time optimal but not cost optimal.

\section{Bounded Number of Processors}

Let us now consider the more practical situation, where the processor number $p$ is arbitrary but fixed: $p<n$, where $p$ divides $n$.

We introduce the type $[\alpha]_{p}$ of lists of length $p$ and use the notation map $_{p}$, etc. for functions defined on such lists. We take the approach from [6]: the input list is distributed over $p$ sublists, which are called blocks. This is done by the distribution function, $\operatorname{dist}(p):[\alpha] \rightarrow[[\alpha]]_{p}$.

The following equality relates distribution with its reverse, flattening:

$$
\operatorname{red}(\#) \circ \operatorname{dist}(p)=i d
$$

Homomorphisms have the following important property.

Theorem 6 (Promotion [1]). For homomorphism h with combine operator $\oslash$ :

$$
h \circ \operatorname{red}(\#)=\operatorname{red}(\oslash) \circ(\operatorname{map} h)
$$

Now, we can start to transform $\oplus \uparrow \otimes$ : 


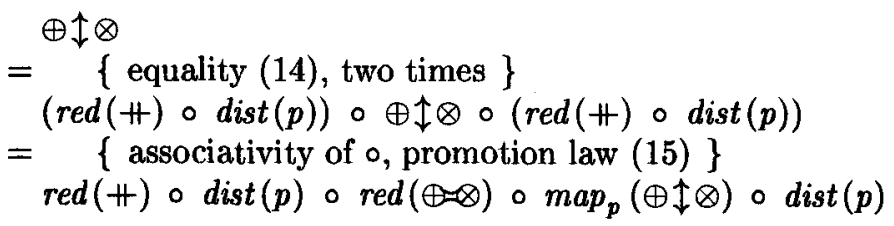

We separate the first, distributing and the last, collecting stage of the result expression, and assume that these stages are implemented by the environment. The remaining, middle part both accepts and yields distributed data of type $[[\alpha]]_{p}$. For an arbitrary function $h$, we call such expression the $p$-distributed version of $h$ and use notation $(\widetilde{h})_{p}$ for it. For a DH, we have:

$$
\widetilde{(\oplus \uparrow \otimes})_{p}=\operatorname{dist}(p) \circ \operatorname{red}(\oplus \otimes) \circ \operatorname{map}_{p}(\oplus \downarrow \otimes)
$$

Program (16) is not optimal: it concatenates and then redistributes the list.

\section{Proposition 7. Redistribution Elimination Rule:}

$$
\operatorname{dist}(p) \circ \operatorname{red}(\oplus \otimes)=((z i p \oplus) \downarrow(z i p \otimes))_{p}
$$

The redistribution elimination (17) applied to (16), together with (12) yields:

$$
\widetilde{(\oplus \uparrow \otimes)_{p}}=\operatorname{swap}_{p}^{k}(z i p(\oplus), z i p(\otimes)) \circ \operatorname{map}_{p}(\oplus \downarrow \otimes)
$$

This is a common $p$-processor implementation of a DH function. It consists of two stages: a sequential computation of the function in all $p$ processors simultaneously on their blocks, and then a sequence of swaps on the hypercube. This implementation has been derived formally and, thus, is provably correct. For an input list of length $n$, the $s w a p$-stage requires $\log p$ steps, with blocks of size $n / p$ to be sent and received and sequential component-wise computations on them; this yields a total time $O((n / p) \cdot \log p)$.

Scan: Efficient Implementation. Since there are scan algorithms with better performance than the general case of $\mathrm{DH}$, we should use some special properties ofscan. The direct specialization of the general program (16) for scan is as follows:

$$
(\widetilde{\operatorname{scan}})_{p}(\odot)=\operatorname{dist}(p) \circ \operatorname{red}(\oslash) \circ \operatorname{map}_{p}(\operatorname{scan}(\odot))
$$

Here, $\oslash$ is from (3). Our goal is to specialize the Redistribution Elimination Rule (17) for the case of such combine operator.

The idea of the transformation is that, instead of performing computations in the blocks at each step of the reduction, we accumulate step-by-step one value for each block and then perform (in one go) the necessary computation across the blocks. For element $a$ and block $u$, the latter computation can be defined as an operation $\odot \triangleleft$, such that $a \odot u=\operatorname{map}(a \odot) u$.

For $\oslash$ of the form (3), the Redistribution Elimination Rule becomes then:

$$
\begin{aligned}
& \left.(\operatorname{dist}(p) \circ \operatorname{red}(\oslash)) x=z i p_{p}(\odot)\right)(y, x), \\
& \quad \text { where } y=\left(\text { prescan }_{p}(\odot) \circ \operatorname{map}_{p}(\text { last })\right) x
\end{aligned}
$$

Here, $z i p_{p}$ is applied to lists of length $p$ and is directly parallelizable like $m a p_{p}$. 
The list $y$ of accumulating values is computed by the prescan function, which yields the result of scan, "shifted to the right":

$$
\operatorname{prescan}(\odot)\left[x_{1}, x_{2}, \cdots, x_{n}\right]=\left[0_{\odot}, x_{1}, x_{1} \odot x_{2}, \cdots, x_{1} \odot x_{2} \odot \cdots \odot x_{n-1}\right]
$$

where $0_{\odot}$ is the neutral element of $\odot$.

Despite its simplicity and analogy to scan, function prescan is not a homomorphism, but it can be adjusted to the DH format exactly like scan, with the only difference in the pairing function, which now is of the form prepair $a=\left(0_{\odot}, a\right)$. A parallel implementation of the prescan function is then of the same three-stage form as the scan implementation (13). After substituting it into (20) and fusing two maps, we obtain from (19) the following target algorithm for $(\widetilde{s c a n})_{p}$ :

$$
\begin{aligned}
(\widetilde{\operatorname{scan}})_{p}(\odot) x & =z_{p} p_{p}(\odot)(y, z) \\
\text { where } z & =\operatorname{map}_{p}(\operatorname{scan}(\odot)) x \\
y & =\left(\operatorname{map}_{p}\left(\pi_{1}\right) \circ \operatorname{swap}_{p}^{k}(\oplus, \otimes) \circ \operatorname{map}_{p}(\text { prepair } \circ \text { last })\right) z
\end{aligned}
$$

with $\oplus, \otimes$ from (11) and $k=\log p$.

Implementation (21) has three stages:

- Compute $z$ : each processor applies the scan function to its block of $x$.

- Compute $y$ : after picking the last elements of their blocks of $z$ and initializing pairs by prepair, the processors work together in $\log p$ swaps; the computations $\oplus$ and $\otimes$ are defined by (11).

- Compute the result: each processor adds (in the sense of $\odot$ ) its element of $y$ to each element of its block of $z$.

This is exactly the known algorithm for scan on a hypercube with an arbitrary fixed number of processors [7]. Its complexity is $O(n / p+\log p)$, which is a clear improvement over the implementation (18) in the general case of DH.

\section{Conclusion}

This paper makes a contribution to parallel programming methodology by giving a definition of the DH class of functions on lists, and a formal derivation of an efficient parallel implementation schema for all functions of the class on a hypercube with either a linear or an arbitrary fixed number of processors. The derivation is based on the semantically sound transformation rules of the BMF, which guarantees its correctness. The performance of the target implementations is easily predictable and conforms with the known estimates.

An argument for the practicality of our approach is that the DH class includes the important scan function and that the specialization of the common implementation yields the parallel algorithm for $s c a n$, which is nowadays considered to be the best in practice [8]. The structure of the parallel implementation for DH is similar to the ascending algorithms of [10], which provides confidence that other important application algorithms can be derived in a similar way. 
Because of the lack of of space, we only mention the related work on divideand-conquer [11], formal derivation of scan algorithms [12, 13, 14], parallelizing transformations in BMF [6] and transition from functional to parallel imperative representations [15]. An extended version of the paper with the full comparison to the related work and other technical details is available in WWW from http://www.brahms.fmi . uni-passau. de/cl/index-gorlatch.

\section{References}

1. R. S. Bird. Lectures on constructive functional programming. In M. Broy, editor, Constructive Methods in Computing Science, NATO ASO Series F: Computer and Systems Sciences. Vol. 55, pages 151-216. Springer Verlag, 1988.

2. G. Blelloch. Scans as primitive parallel operations. IEEE Trans. on Computers, 38(11):1526-1538, November 1989.

3. J. Misra. Powerlist: a structure for parallel recursion. ACM TOPLAS, 16(6):1737$1767,1994$.

4. S. Gorlatch. Stages and transformations in parallel programming. In M. Kara et al., editors, Abstract Machine Models for Parallel and Distributed Computing, pages 147-162. IOS Press, 1996.

5. S. Gorlatch. Constructing list homomorphisms. Technical Report MIP-9512, Universität Passau, 1995.

6. D. Skillicorn and W. Cai. A cost calculus for parallel functional programming. Journal of Parallel and Distributed Computing, 28:65-83, 1995.

7. M. J. Quinn. Parallel Computing. McGraw-Hill, Inc., 1994.

8. M. Reid-Miller. List ranking and list scan on the Cray C-90. In Proceedings SPAA'94, pages 104-113, 1994.

9. D. Walker. The design of a standard message passing interface for distributed memory concurrent computers. Parallel Computing, 20:657-673, 1994.

10. F. Preparata and J. Vuillemin. The cube-connected cycles: A versatile network for parallel computation. Communications of the ACM, 24(5):300-309, 1981.

11. Z. G. Mou. Divacon: A parallel language for scientific computing based on divide and conquer. In Proc. 3rd Symposium on the Frontiers of Massively Parallel Computation, pages 451-461, October 1990.

12. J. O'Donnell. A correctness proof of parallel scan. Parallel Processing Letters, 4(3):329-338, 1994.

13. J. Gibbons. Upwards and downwards accumulations on trees. In R. Bird, C. Morgan, and J. Woodcock, editors, Mathematics of Program Construction, Lecture Notes in Computer Science 669, pages 122-138, 1992.

14. J. Kornerup. Mapping a functional notation for parallel programs onto hypercubes. Information Processing Letters, 53:153-158, 1995.

15. K. Achatz and W. Schulte. Architecture independent massive parallelization of divide-and-conquer algorithms. In B. Moeller, editor, Mathematics of Program Construction, Lecture Notes in Computer Science 947, pages 97-127, 1995. 